\title{
Journal of Gastroenterology Research
}

ISSN: $2578-6210$

Review Article

DOI: $10.36959 / 621 / 626$

\section{Trials of Meridian Therapies, Such as Acupuncture, Moxibustion, Hijama and Marma, in the Management of Irritable Bowel Syndrome: A Review of the Limitations}

\author{
Affifa Farrukh, MB MSc, LLM MRCP, FRCP FRCPI* \\ Department of Digestive Diseases, Leicester General Hospital, Gwendolen Road, Leicester, LE5 4PW, \\ United Kingdom
}

\begin{abstract}
This review considers the role of meridian therapies, such as acupuncture, moxibustion, marma and hijama, in treatment of patients with irritable bowel syndrome (IBS) and the difficulties of designing clinical trials to assess their effectiveness. The issue of distinguishing case from non-case because of a lack of a uniform and consistent definition of the disease together with choice of appropriate placebos are analysed and the "rituals of healing" associated with such therapies considered.
\end{abstract}

Keywords

Meridian therapies, Acupuncture, Moxibustion, Marma, Hijama, Irritable bowel syndrome

\section{Introduction}

Current allopathic treatments for irritable bowel syndrome (IBS) are of limited efficacy and directed at symptom control. This paper will review readily and freely accessible published and data work in the public domain. For example, a Cochrane Review demonstrated no benefit from bulking agents or antidepressants in pain reduction. [1]. However, a sub-group analysis confirmed some benefit from peppermint oil. Rees, et al. [2] developed this traditional herbal remedy because conventional drugs were "often disappointing and their use limited by side effects". Traditional and complementary therapies were taken up by other researchers. Whorwell, et al. [3] reported a comparative study of psychotherapy and hypnotherapy.

Patients who received hypnotherapy showed a highly significant improvement in their symptoms of abdominal pain, bloating, bowel frequency and general well-being which was maintained for three months of follow-up. Patients treated with psychotherapy also showed an improvement, but its degree was significantly less than with hypnotherapy. The role of biofeedback as a potential therapy also emerged at this time, although studies were small [4]. Complementary therapies have been used as treatment for IBS by around $41 \%$ patients, with homeopathic remedies the most popular followed by acupuncture [5]. In a more recent Dutch study of 750 children, almost $40 \%$ of parents had sought a complementary consultation and $22 \%$ were on such therapy as a result [6].

\section{Acupuncture and Related Therapies}

The classical theory underlying acupuncture concerns circulation of qi through the ching-lo system and its way stations [7]. These way-stations or acupoints are located along 12 meridians which run the length of the body. Disease arises when there is disharmony and this disharmony can be linked to the environment, emotional outlook and way of life [8].

Disharmony results in an obstruction to flow of qi along the meridians. The purpose of treatment is to eliminate disharmony and bring the system back into balance. The underlying philosophy is that stimulation of various acupuncture points along the meridians can achieve this. Traditional Chinese needles are said to be rather rough and are pushed deeper, rotated and moved up and down by

*Corresponding author: Affifa Farrukh, MB MSc (Pain Management), LLM MRCP (Gastroenterology) FRCP FRCPI, Consultant Physician, Gastroenterologist \& Barrister (Non-Practicing), Department of Digestive Diseases, Leicester General Hospital, Gwendolen Road, Leicester, LE5 4PW, United Kingdom

Accepted: December 29, 2021

Published online: December 31, 2021

Citation: Farrukh A (2021) Trials of Meridian Therapies, Such as Acupuncture, Moxibustion, Hijama and Marma, in the Management of Irritable Bowel Syndrome: A Review of the Limitations. J Gastroenterol Res 5(2):224-228 
Citation: Farrukh A (2021) Trials of Meridian Therapies, Such as Acupuncture, Moxibustion, Hijama and Marma, in the Management of Irritable Bowel Syndrome: A Review of the Limitations. J Gastroenterol Res 5(2):224-228

practitioners with the intention of creating a "De qi" sensation which may be perceived as numbness, heaviness or tingling. This feeling is believed to be associated with unblocking the meridian. However, in Japanese acupuncture the needles used are thin, inserted superficially and not rotated. In Korea many practitioners use only 4 needles; two to sedate or reduce excess $Q i$ in one organ system and the other two to increase $Q i$ in a second organ system and so eliminate imbalance or disharmony [9]. Dr. Ching-Chang Tung in Taiwan concentrated on use of peripheral points, particularly in the arms and leg, and this practice is becoming widespread in the West [10].

In a study of 30 patents with IBS Chu, et al. [11] investigated the impact of electro-acupuncture on pain caused by experimental rectal distension. True electroacupuncture led to significantly higher activation at the right insula, and the pulvinar and medial nucleus of the thalamus compared to sham. They suggested that acupuncture might modulate pain in IBS through effects on the serotonin pathway at the level of the insula and so improve patients' mood via the ascending pathway at the pulvinar and medial nucleus of the thalamus. Work from Michigan, using positron emission tomography (PET) with [11] C-carfentanil, has shown long-term increases in mu-opioid receptors binding potential in the cingulate, caudate, and amygdala of patients receiving traditional acupuncture compared to sham and this was associated with greater reductions in pain experience [12]. Such studies are beginning to define how acupuncture may affect the physiological mechanisms responsible for pain.

Acupuncture, moxibustion, marma and hijama share a number of features with treatment applied at similar points on the body. Acupuncture and moxibustion are routinely used together by traditional Chinese practitioners for management of IBS, although in western practice they are often prescribed separately. Hijama is also routinely advertised on the internet as an effective treatment for IBS [13], while marma forms an integral part of the Ayurvedic holistic approach to management of IBS. None of these therapies are regulated in the UK. Nevertheless, millions of acupuncture treatments are given annually in the UK [14]. However, there are no data for the frequency with which moxibustion, hijama or marma treatments are administered.

\section{Acupuncture}

In 1962, Acupuncture, The Ancient Chinese Art of Healing, referred to its use for the treatment of IBS and related conditions:

"Constipation, diarrhoea, various types of colic, and atony" [15].

\section{The commonest approaches include:}

1. Treating Liver qi stasis by needling Liver 3 and Large Intestine 4 acupoints which relieves wind.

2. Spleen qi deficiency is treated by needling Stomach 36 and Spleen 4.

3. Needling abdominal points such as Stomach 25 and Spleen 15 can alleviate diarrhoea or constipation.
4. Pericardium 6 may be need led to help with stress as might Spleen 9.

In an individual case, some or all of these points might be needled as well as additional points. For each patient a specific protocol will be developed. The significance of such a wide range of points and use of different needling techniques as well as individualised treatment for each patient have direct consequences when considering trial design and analysis in the treatment of IBS, a condition where the diagnosis itself can vary from practitioner to practitioner. Further, Choi and Tweed [16] have shown that people's perceptions of acupuncture were affected by both the style and gender of practitioners. Male holistic practitioners were considered the most effective therapists, suggesting that detailed discussions about a patient's health and wide-ranging advice had a significant impact compared to simply placing needles at appropriate points. There was also evidence that the more attractive the therapist, regardless of gender, the more effective they were considered by the assessors! Choi and Tweed [16] were also able to show that the combination of acupuncture together with moxibustion was most effective, when administered in a holistic setting. The importance of patients' beliefs and expectations has been further confirmed in a PET scanning study which showed that expectation of a real effect from sham acupuncture caused greater activation than simple pin prick, although less so than real acupuncture [17]. This confirmed the role of patients' perceptions and the potential impact of an attractive holistic therapist on how treatment might be regarded.

\section{Moxibustion}

Moxa is made from the dried leaves of Artemisia vulgaris and usually formed into small pyramids or cigar shaped rolls. When lit it smoulders and the heat is placed either on (direct) or close (indirect) to acupuncture points until discomfort is felt. If left longer there can be blistering and scarring. In traditional practice this was considered the most effective form of therapy, but is clearly unacceptable in Western practice.

Burning moxa produces a radiation spectrum ranging from 0.8 to $5.6 \mu \mathrm{m}$; with a peak near $1.5 \mu \mathrm{m}$, and so lying within the near infrared portion [18]. However, its effects may also be due to inhalation of moxa smoke, which is a characteristic product of traditional therapy in contrast to some modern smokeless moxas. There has been one MRI based study of moxibustion in IBS. Moxa abolished activation of the prefrontal cortex and the anterior cingulated cortex in response to rectal distension, while a control group showed no such effect [19].

\section{Hijama}

Hiiama is a form of wet cupping. Suction cups are applied to acupuncture points and an area of reddened skin raised. Superficial small incisions are made on this skin and cups re-applied to draw up blood into the cup. During the last decade there has been a dramatic growth in the practice of Hijama across the USA and the UK [20]. Although both dry and wet cupping were widely practiced, Hijamais now 
Citation: Farrukh A (2021) Trials of Meridian Therapies, Such as Acupuncture, Moxibustion, Hijama and Marma, in the Management of Irritable Bowel Syndrome: A Review of the Limitations. J Gastroenterol Res 5(2):224-228

largely associated with Muslim communities and offered by untrained practitioners or those who have completed spurious educational programs [20]. Of 27 papers identified through Pubmed, using the search term "Hijama", only six reported clinical trials and as a result there is limited academic information available on its efficacy.

\section{Marma}

Marma may be considered the Ayurvedic equivalent of acupuncture. As with the meridians of acupuncture, nadis form an interconnected network throughout the body [21]. Unlike meridians, nadis are not mapped on the surface but as a visualisation of internal pathways connecting structures throughout the body. They can only be influenced by massage or heat applied to energy points or marmani and this can have both distant and local effects. Marma points are larger in area than the more discrete acupuncture points and fewer in number with only 60 in regular use [22]. Despite its long history, there have been few studies of the benefits of Marma therapy using accepted trial design, although there is some limited evidence supporting its use in stroke rehabilitation [23].

\section{The Design of Clinical Trials in IBS using Acupuncture and Related Therapies}

Randomised control double blind studies are generally accepted as the type of study which provides adequate control for bias and sound evidence of cause and effect. This type of study would be the most suitable to measure the effect of traditional techniques, such as acupuncture, considering the complexity of IBS and the difficulties that might arise. The trial should be designed to answer a clear question and whether the treatment is comparable or superior to the other treatments or a placebo.

\section{Important Elements for Trials in IBS}

Patients in an IBS trial need a clear and certain diagnosis. They should be representative of the population from which they are drawn, and ideally include ethnic minorities. In addition, the study should include sufficient patients to ensure it is adequately powered to provide a reliable answer to the question asked. Outcome measures need to be valid and reliable. Changes in symptoms and their impact on daily life are the only outcomes which can be reliably assessed in IBS. Of symptoms, pain is the most consistent, while its impact on time lost from work and resulting depression are all features which could be assessed either by an external observer or through patient reported outcomes. The issue of bias can be addressed through randomisation and blinding of external assessors.

\section{Design of Trials in IBS}

Within the study design the control group can be one of six types according to the Food and Drug Administration classification namely: Placebo concurrent controls, activetreatment concurrent controls, no-treatment concurrent controls, dose-comparison concurrent controls, external controls and multiple controls [24]. Pharmacological treatments, such as mebeverine and peppermint oil, are in regular use in IBS and so the most suitable study design should have active-treatment concurrent controls. However, the comparison of tablet therapy with an intervention which includes significant interaction between patient and therapist may limit an assessment of the technique itself. Therefore, the introduction of a placebo concurrent control for an intervention, such as acupuncture, is needed. However, there is an ethical issue related to use of placebos where an effective alternative treatment exists. Under Article 32 of the Declaration of Helsinki such an approach can be acceptable where for sound methodological reasons use of placebo is necessary to determine the efficacy of an intervention and patients who receive placebo will not be subject to any risk of serious harm [25].

The importance of "blinding" was underlined by Day \& Altman [26]. It is particularly important when the response criteria are subjective, such as relief of pain, which is the main outcome measure in IBS trials and is usually reported by patients. Patients are seldom blind to an active intervention, even when a sham treatment has been used. Schulz, et al. [27] have shown that the effect of inadequate concealment is a significantly larger estimate of treatment effect. In the case of interventions, such as acupuncture or moxibustion, concealment from patients is almost impossible.

The specific issues, in relationship to assessment of trials investigating the efficacy of acupuncture and related therapies in the management of IBS include assessments of:

1. The diagnostic criteria used to identify subjects for the trial.

2. The method of selection of patients from amongst suitable subjects for inclusion in the trial.

3. Randomisation procedures and whether they are transparent.

4. The nature of the placebo.

5. The style of acupuncture and type of moxa used.

6. The skills of the practitioners.

7. Details of blinding of patients, practitioners and assessors.

8. The outcome measures used and whether validated.

9. Duration of the trial and follow-up.

10. Number of participants and whether a power calculation was performed prior to initiation of the trial.

11. Drop-out rates.

12. Potential bias amongst authors of the publication.

Based on Evans \& Ilstad [24] and the Food and Drug Administration [28].

\section{The Diagnostic Criteria used to Identify Subjects for the Trial}

The diagnosis of IBS is based on symptoms as there 
Citation: Farrukh A (2021) Trials of Meridian Therapies, Such as Acupuncture, Moxibustion, Hijama and Marma, in the Management of Irritable Bowel Syndrome: A Review of the Limitations. J Gastroenterol Res 5(2):224-228

are no objective laboratory-based tests which can identify patients. The critical symptoms for reaching a clinical diagnosis have been defined in two commonly used tools, namely the Manning [29] and Rome [30] criteria. The Rome criteria have gone through several revisions with Rome IV being the latest. Unfortunately, Manning and Rome criteria demonstrate significant differences when distinguishing case from non-case. In a comparative study using the criteria proposed by Manning and Rome I and II the prevalence of IBS in a community based study of 5000 people was reported as $16.2 \%$, (Manning with only 2 symptoms) $9.7 \%$, (Manning with 3 symptoms) 5.6\%, (Rome I) and 5.1\% (Rome II)., Ford, (2008) demonstrated that the diagnostic value of Manning criteria was only modest in a systematic review of 10 studies involving 2355 patients [31].

\section{The Nature of Controls in IBS Studies}

In 2007 Dorn, et al. reviewed 19 trials of complementary therapy in IBS where the placebo response rate was $42.6 \%$ (95\% Cl 38.0-46.5\%) [32]. The placebo response rate did not correlate with type of treatment or trial duration. However, it did correlate with duration of treatment and number of office visits. Such findings emphasised Vincent and Lewith's call for credible controls in any investigation of acupuncture and the potential impact of the "holistic" approach to care [33]. Support for such a view also comes from a systematic review of the placebo effect of psychological interventions in the treatment of IBS, which had comparable response rates [34].

\section{Conclusions}

The creation of a credible placebo for acupuncture, moxibustion, massage and other complementary interventions is a significant challenge for researchers. Dorn, et al. [32] consider that the holistic approach taken by practitioners of complementary therapy cannot easily be separated into its component parts and as Kaptchuk [35] has suggested the "healing ritual" associated with such therapies may be a significant factor in their clinical outcomes. These factors are seldom reproduced in clinical trials and if they are may enhance the response to a placebo control.

\section{References}

1. Ruepert L, Quartero AO, de Wit NJ, et al. (2011) Bulking agents, antispasmodics and antidepressants for the treatment of irritable bowel syndrome. Cochrane Database Syst Rev 8: CD003460.

2. Rees WD, Evans BK, Rhodes J (1979) Treating irritable bowel syndrome with peppermint oil. Br Med J 2: 835-836.

3. Whorwell PJ, Prior A, Faragher EB (1984) Controlled trial of hypnotherapy in the treatment of severe refractory irritablebowel syndrome. Lancet 2: 1232-1234.

4. Radnitz CL, Blanchard EB (1989) A 1- and 2-year follow-up study of bowel sound feedback as a treatment of irritable bowel syndrome. Biofeedback Self Regul 14: 333-338.

5. Smart HL, Mayberry JF, Atkinson M (1986) Alternative medicine consultations and remedies in patients with the irritable bowel syndrome. Gut 27: 826-828.

6. Vlieger AM, Blink M, Tromp E, Benninga MA (2008) Use of complementary and alternative medicine by pediatric patients with functional and organic gastrointestinal diseases: Results from a multicenter survey. Pediatrics 122: e446-e451.

7. Lu GW, Needham J (1980) Celestial Lancets. A History and Rationale of Acupuncture and Moxa. Routledge, London.

8. Kaptchuk T (1983) Chinese Medicine. The Web that has no Weaver.

9. National University of Health Sciences (2014) Korean, Japanese and Chinese acupuncture: What's the difference?

10. Lee M (1992) Insights of a Senior Acupuncturist: One Combination of Points can Treat Many Diseases. Blue Poppy Press, Boulder, USA.

11. Chu WC, Wu JC, Yew DT, et al. (2012) Does acupuncture therapy alter activation of neural pathway for perception in irritable bowel syndrome? a comparative study of true and sham acupuncture using functional magnetic resonance imaging. J Neurogastroenterol Motil 18: 305-316.

12. Harris RE, Zubeita JK, Scott DJ, et al. (2009) Traditional Chinese acupuncture and placebo (sham) acupuncture are differentiated by their effects on mu-opiod receptors (MORs). Neuroimage 47: 1077-1085.

13. Lemu M (2017) Hijama (Cupping) for irritable bowel syndrome.

14. Hopton A, Curnoe S, Kanaan M, et al. (2011) Acupuncture in practice: Mapping the providers, the patients and the settings in a national cross-section survey. BMJ Open 2: e000456.

15. Felix M (1971) Acupuncture The Ancient Chinese Art of Healing. William Heinemann Medical Books Ltd, London.

16. Choi PY, Tweed A (1996) The holistic approach in acupuncture treatment: Implications for clinical trials. J Psychosom Res 41: 349-356.

17. Pariente J, White P, Frackowiak RS, et al. (2005) Expectancy and belief modulate the neuronal substrates of pain treated by acupuncture. Neuroimage 25: 1161-1167.

18. Deng H, Shen X (2013) The mechanism of moxibustion: Ancient theory and modern research. Evid Based Complement Alternat Med 2013: 379291.

19. Zhu Y, Wu Z, Ma X, et al. (2014) Brain regions involved in moxibustion-induced analgesia in irritable bowel syndrome with diarrhea: A functional magnetic resonance imaging study. BMC Complement Altern Med 14: 500.

20. Mayberry JF (2017) Clinically applied Hijama therapy in the United Kingdom: The need for statutory regulations. J Muslim Minor Aff 37: 321-331.

21. Lad V, Durve A (2015) Marma Points of Ayurveda. The Ayurvedic Press, Alburquerque, Mexico.

22. Frawley D, Ranade S, Lele A (2003) Ayurveda and Marma Therapy. Lotus Press Twin Lakes.

23. Sankaran R, Kamath R, Nambiar V, et al. (2018) A prospective study on the effects of Ayurvedic massage in post-stroke patients. J Ayurveda Integr Med 10: 126-130.

24. Food and Drug Administration (2001) Guidance for industry e10 choice of control group and related issues in clinical trials.

25. (2013) Declaration of Helsinki.

26. Day SJ, Altman DG (2000) Blinding in clinical trials and other studies. BMJ 321: 504.

27. Schulz KF, Chalmers I, Hayes RJ, et al. (1995) Empirical evidence 
Citation: Farrukh A (2021) Trials of Meridian Therapies, Such as Acupuncture, Moxibustion, Hijama and Marma, in the Management of Irritable Bowel Syndrome: A Review of the Limitations. J Gastroenterol Res 5(2):224-228

of bias. Dimensions of methodological quality associated with estimates of treatment effects in controlled trials. JAMA 273: 408-412.

28. Evans CH, Ildstad ST (2001) Design of small clinical trials In Smal Clinical Trials: Issues and Challenges. Institute of Medicine (US) Committee on Strategies for Small-Number-Participant Clinical Research Trials, National Academies Press, Washington, USA.

29. Manning AP, Thompson WG, Heaton KW, Morris AF (1978) Towards positive diagnosis of the irritable bowel. Br Med J 2: 653-654.

30. Drossman DA, Hasler WL (2016) Rome IV- Functional gastrointestinal disorders. Disorders of Gut-Brain Interaction. Gastroenterology 150: 1257-1261.

31. Ford AC, Talley NJ, Veldhuyzen van Zanten SJO, et al. (2008) Will the history and physical examination help establish that irritable bowel syndrome is causing this patient's lower gastrointestinal tract symptoms?. JAMA 300: 1793-1805.
32. Dorn SD, Kaptchuk TJ, Park JB, et al. (2007) A meta-analysis of the placebo response in complementary and alternative medicine trials of irritable bowel syndrome. Neurogastroenterol Motil 19: 630-637.

33. Vincent C, Lewith G (1995) Placebo controls for acupuncture studies. J R Soc Med 88: 199-202.

34. Filk CE, Bakker L, Laan W, et al. (2017) Systematic review: The placebo effect of psychological interventions in the treatment of irritable bowel syndrome. World Journal of Gastroenterol 23: 2223-2233.

35. Kaptchuk TJ (2002) The placebo effect in alternative medicine: Can the performance of a healing ritual have clinical significance? Ann Inter Med 136: 817-825. 